\title{
Modulation of Transient Receptor Potential (TRP) channels by tyrosine phosphorylation
}

\section{Alexandra Manolache, Teodora Stratulat, Alexandru Babeș ${ }^{\bowtie}$}

\author{
Department of Anatomy, Physiology and Biophysics, Faculty of Biology, University of Bucharest, Bucharest, \\ Romania \\ ${ }^{\bowtie}$ Correspondence: alexandru.babes@bio.unibuc.ro
}

Received: 8 December 2020 / Revised: 5 February 2021 / Accepted: 11 February 2021 / Available online: 2 March 2021

\begin{abstract}
Transient Receptor Potential (TRP) channels are a superfamily of polymodal, non-selective receptors, expressed in the nervous system and several other tissues, where they play many physiological or pathological roles. TRP channels are sensitive to a diverse range of stimuli, such as temperature, osmolarity, oxidative stress, external compounds and intracellular signaling molecules. The activity of TRP channels can be modulated by protein phosphorylation, including tyrosine phosphorylation. In this review, we present the studies carried out so far regarding the modulation of TRP channels by tyrosine phosphorylation.
\end{abstract}

Keywords: TRP channels, tyrosine phosphorylation, channel activity

\section{Introduction}

Transient Receptor Potential (TRP) channels comprise a superfamily of non-selective ion channel which account for a variety of physiological roles, acting in most cases as sensor of the extracellular environment. The 28 mammalian TRP channels are organized in 6 subfamilies: canonical (TRPC, 7 isoforms), melastatin (TRPM, 8 isoforms), vanilloid (TRPV, 6 isoforms), ankyrin (TRPA, one isoform), polycystin (TRPP, 3 isoforms) and mucolipin (TRPML, 3 isoforms) (Cohen and Moiseenkova-Bell, 2014). These channels are characterized by various gating mechanisms, responding to intracellular signaling molecules (e.g., diacyl glycerol (TRPC3) (Hofmann et al., 1999) or calcium ions (TRPM5) (Liu and Liman, 2003), changes in ambient temperature (e.g., noxious heating (TRPV1) (Caterina et al., 1997), innocuous cold (TRPM8) (McKemy et al., 2002) or oxidative stress (e.g., TRPM2 and TRPA1) (Hara et al., 2002; Andersson et al., 2008). Moreover, they have different cation permeabilities, ranging from being permeable purely to monovalent cations (e.g., TRPM4 and TRPM5) (Guinamard et al., 2011) to being substantially more permeable to divalent cations (e.g., TRPV5 and TRPV6) (Peng et al., 1999; Vennekens et al., 2000). Modulation by cellular signaling pathways is a hallmark of all TRP channels, and the alteration of their biophysical features following the activation of various signaling cascades has a profound effect on the physiology and pathophysiology of these channels.
In this review we shall focus on tyrosine phosphorylation, an important mechanism of channel modulation and less investigated compared to serine/threonine phosphorylation, at least as far as TRP channels are concerned.

\section{The Canonical Transient Receptor Potential ion channels}

The Canonical Transient Receptor Potential (TRPC) proteins are non-selective cation permeable channels, responsible for changes in the cytosolic free $\mathrm{Ca}^{2+}$ concentration (Birnbaumer et al., 1996). The TRPC family comprises seven ion channels, although TRPC2 is a pseudogene in humans (Liman and Innan, 2003). Based on sequence homology and functional similarities, human TRPC channels are grouped in two subfamilies, TRPC1, 4, 5 and TRPC3, 6, 7 (Hofmann et al., 2002). All TRPC channels have six transmembrane domains, intracellularly located $\mathrm{N}$ - and $\mathrm{C}$-terminal regions and a channel pore which is located between transmembrane domains 5 and 6 (Vannier et al., 1998). The N-terminal region possesses ankyrin repeats, a coiled-coil domain, and a putative caveolin-binding region, while the TRP motif and the calmodulin/inositol 1,4,5-trisphosphate receptor-binding region are located in the C-terminal region (Vazquez et al., 2004a). A unique motif to TRPC4 and TRPC5 is the PDZ-binding motif which can interact with cytoskeletal scaffolding proteins (Mery et al., 2002). 
It has been clearly established that the main role of the TRPC ion channels is to mediate receptor-operated and store-operated $\mathrm{Ca}^{2+}$ entry (Clapham et al., 2001). Growth factors, hormones and neurotransmitters bind to $G$ protein-coupled receptors which activate phospholipase $\mathrm{C}$ (PLC), leading to inositol 1,4,5-trisphosphate and diacylglycerol (DAG) formation. It was shown that DAG can activate TRPC $3 / 6 / 7$ channels (Okada et al., 1999), while the TRPC1/4/5 subfamily is activated through more complicated pathways, which may involve other interacting proteins and post-translational modifications (Liu et al., 2020). Several studies have explored the posttranslational modifications and mutations of TRPCs which may modulate the activity of the channels.

\section{TRPC3}

The human isoform of TRPC3, first cloned by Zhu at al. (Zhu et al., 1996), is activated by DAG and DAG homologues, such as 1-oleoyl-2-acetyl-snglycerol (OAG) (Hofmann et al., 1999). The possible mechanism underlying this activation is the receptor-mediated activation of PLC, independently of inositol 1,4,5trisphosphate or $\mathrm{Ca}^{2+}$ store depletion. Tyrosine phosphorylation is a common modification associated with the heterotrimeric $\mathrm{G}$ protein $\mathrm{Gq}-\mathrm{PLC}-\mathrm{Ca}^{2+}$ signaling pathway (Gutkind and Robbins, 1992; Igishi and Gutkind, 1998). The non-receptor tyrosine kinase Src plays a significant role in TRPC3 activation. Using various modalities to inhibit the Src kinase - pretreatment of TRPC3-expressing cells with Src kinase inhibitors, TRPC3-HEK293 cell transfection with a dominantnegative form of Src and expression of TRPC3 into SYF cells $\left(\mathrm{Src}^{--}\right.$, $\mathrm{Yes}^{-{ }^{-}}$and $\left.\mathrm{Fyn}^{--}\right)$- Vazquez et al. showed the key role of Src kinase in TRPC3 activation, as the inhibition of Src kinase suppressed both receptor- and OAG-dependent activation of TRPC3. A putative mechanism underlying this effect is based on a concerted role of DAG and Src kinase, which would involve phosphorylation by Src kinase and/or recruitment of an unknown regulatory protein (Vazquez et al., 2004b). Based on these results, another team extended the investigation on Src-mediated TRPC3 activation and, using a coimmunoprecipitation assay of COS-7 cells, demonstrated a direct interaction between Src kinase and TRPC3. Moreover, using site-directed mutagenesis experiments, they identified four phosphorylated tyrosine residues, Y49, Y148, Y150, and Y226, although just phospho-Y226 was crucial for the activation of TRPC3. Another important result was that neither Yes nor Fyn kinases, other members of the Src kinase family, could induce TRPC3 activation. Interestingly, Y226 is a conserved residue between TRPC3, TRPC6 and TRPC7, but the cognate Y226 did not modify the activation of TRPC6 or TRPC7 (Kawasaki et al., 2006).

\section{TRPC4}

TRPC4 was first cloned by Philipp et al. and was reported to be activated by inositol 1,4,5-trisphosphate or thapsigargin-induced store depletion when expressed in mammalian cells (Philipp et al., 1996). Compared with store-operated channels or other TRPCs, TRPC4, as well as TRPC5, can be potentiated by lanthanides (Schaefer et al., 2000). TRPC4 and TRPC5 contain a unique motif (PDZ - VTTRL) in the carboxy terminal region which can interact with scaffolding proteins, for example the $\mathrm{Na}^{+} / \mathrm{H}^{+}$exchanger regulatory factor (NHERF). This interaction is important for the insertion and maintenance of TRPC4 channels at the plasma membrane (Mery et al., 2002). TRPC4 is an important component of storeoperated $\mathrm{Ca}^{2+}$ channels. Experiments on TRPC4 null mouse demonstrated the essential role of TRPC4 in the calcium regulation of aortic endothelial cells (Freichel et al., 2001). Stimulation of epidermal growth factor (EGF) receptor in corneal epithelial cells leads to the activation of a store-operated current mediated by TRPC4 (Yang et al., 2005). Further studies showed that human TRPC4 expressed in COS-7 cells is phosphorylated at Y959 and Y972 tyrosine residues in the C-terminus after EGF receptor stimulation, and this effect is due to the $\mathrm{Src}$ family non-receptor tyrosine kinases, especially Fyn kinase. The phosphorylation of hTRPC4 increases the association between TRPC4 and NHERF which facilitates the insertion of TRPC4 into plasma membrane, which leads to an increase of $\mathrm{Ca}^{2+}$ influx. Mutation of Y959 and Y972 to phenylalanine impaired the TRPC4NHERF association and subsequent translocation of TRPC4 (Odell et al., 2005).

\section{TRPC6}

TRPC6 is a cation channel more permeable to $\mathrm{Ca}^{2+}$ than to $\mathrm{Na}^{+}$, which can form homo- and heterotetramers in vivo (Hofmann et al., 1999). Mouse TRPC6 was isolated from the brain (Boulay et al., 1997) and human TRPC6, which shares $93 \%$ amino acid identity with the murine channel, was first cloned in 1999 from the placenta (Hofmann et al., 1999). Like TRPC3, TRPC6 can be activated by DAG and DAG analogue OAG, independently of protein kinase C (Hofmann et al., 1999). A mechanism for TRPC6 channel modulation is direct tyrosine phosphorylation induced by Src protein tyrosine kinases. Hisatsune et al. found that Fyn and Src kinases can phosphorylate TRPC6 in heterologous expression systems. In COS-7 cells, Fyn physically interacts with the N-terminal part of TRPC6 via the $\mathrm{SH} 2$ domain. Moreover, the association of TRPC6 with Fyn kinase could be observed in the rat brain. Similar to the results of Odell et al. concerning TRPC4 (Odell et al., 2005), TRPC6 tyrosine phosphorylation can be induced via EGF receptor stimulation in COS-7 cells and the phosphorylation is abolished by treatment with PP2, a specific inhibitor of Src family kinases, or by a dominant negative form of Fyn. The functional consequence of 
TRPC6 tyrosine phosphorylation was observed using the inside-out patch clamp recording technique. TRPC6 activity was increased following application of Fyn and adenosine triphosphate to the cytoplasmic face of membrane patches excised from TRPC6-expressing cells. Thus, Fyn-mediated tyrosine phosphorylation enhances TRPC6 channel activity, which leads to an increased calcium influx (Hisatsune et al., 2004). An effect of TRPC6 tyrosine phosphorylation was observed in mouse glomerular epithelial cells (podocytes). Stimulation of mouse podocytes with transforming growth factor beta1 (TGF- $\beta 1$ ) increases the expression of TRPC6 and Fyn kinase, especially the active form of the enzyme (Fyn pY418), leading to an increase in TRPC6 tyrosine phosphorylation. Importantly, the cytosolic free $\mathrm{Ca}^{2+}$ level, reflecting TRPC6 activity, was significantly elevated after TGF- $\beta 1$ treatment in TRPC6overexpressing cells. Moreover, TRPC6 overexpression notably aggravated podocyte apoptosis induced by TGF$\beta 1$, while TRPC6 knockdown inhibited TGF- $\beta 1$-induced podocyte apoptosis (Yu et al., 2010). Following this line of investigation, another group studied TRPC6 phosphorylation in HEK293T cells and in cultured mouse podocytes and found that phosphorylation of Y284 is involved in Fyn-induced membrane expression of TRPC6 (Kanda et al., 2011). Moreover, PLC- $\gamma 1$ binds to Y31 and Y284 and this interaction is crucial for TRPC6 membrane trafficking. Interestingly, nephrin, an essential protein component of the podocyte slit diaphragm, interacts with the phosphorylated domain of TRPC6 and reduces channel surface expression, an opposite effect to that of PLC $-\gamma 1$. In this study it was proposed that in patients with focal segmental glomerulosclerosis, mutations of TRPC6 can reduce the contact with nephrin and promote the interaction with PLC- $\gamma 1$, leading to an increase in TRPC6 expression and activity in the podocytes (Kanda et al., 2011). Other studies have shown the upregulation of TRPC6 channels in podocytes caused by an increased generation of reactive oxygen species (Kim et al., 2012, Anderson et al., 2014). In a recent study it was shown that soluble urokinase and plasminogen activator receptor induces reactive oxygen species generation in mouse podocytes via Nox 2 complex that activate Src family kinases, leading to the upregulation of TRPC6 channels. The effects of oxidative stress and altered $\mathrm{Ca}^{2+}$ signaling may contribute to podocyte apoptosis and disease progression in focal segmental glomerulosclerosis (Kim et al., 2018).

\section{The Melastatin Transient Receptor Potential ion channels}

The Melastatin Transient Receptor Potential subfamily was named after the first described member, TRPM1, a putative tumor suppressor protein (Duncan et al., 1998). The TRPM subfamily includes eight members, classified in four groups based on their structural homology: TRPM1/3, TRPM6/7, TRPM4/5, and TRPM2/8 (Zholos et al., 2011). TRPM members are non-selective cation permeable channels, but only TRPM6 and TRPM7 are $\mathrm{Mg}^{2+}$-permeable and thus involved in the regulation of intracellular $\mathrm{Mg}^{2+}$ (Li et al., 2006). Furthermore, TRPM6 and TRPM7 contain an active $\alpha$-kinase domain in the $\mathrm{C}$ terminal region, allowing them to function as "chanzymes" (Montell, 2003). The TRPM family includes several channels involved in cell proliferation or survival, such as TRPM2 (Fonfria et al., 2005; Zhang et al., 2006), TRPM5 (Prawitt et al., 2000), TRPM7 (Aarts et al., 2003) and TRPM8 (Tsavaler et al., 2001). TRPM8 is the only temperature-sensitive channel of this subfamily (McKemy et al., 2002). Like most TRP channels, TRPM members are modulated by posttranslational modifications, although the number of reports on the modulatory role of tyrosine phosphorylation on TRPM channels is limited.

\section{TRPM2}

TRPM2, the second TRPM member described (Harteneck et al., 2000), is a $\mathrm{Ca}^{2+}$-permeable channel expressed in various tissues, for example the brain and hematopoietic cells (Sano et al., 2001; Hara et al., 2002). TRPM2 is activated by oxidative stress, tumor necrosis factor $\alpha(\mathrm{TNF} \alpha)$, amyloid $\beta$-peptide and concanavalin A (Hara et al., 2002; Wehage et al., 2002; Fonfria et al., 2005; Gasser et al., 2006; Zhang et al., 2006). The mechanism of activation involves the production of intracellular ADP ribose which binds to the C-terminal region of TRPM2 leading to channel activation and elevation of free intracellular calcium concentration (Wehage et al., 2002; Gasser et al., 2006). Thus, TRPM2 plays a crucial role in oxidative stress-induced or TNFa activation-induced cell apoptosis (Hara et al., 2002; Fonfria et al., 2005; Zhang et al., 2006). A more in-depth study revealed that tyrosine phosphorylation is an important post-translational modification of TRPM2, that regulates its activation and function. It has been shown that by inhibiting tyrosine phosphorylation of TRPM2, $\mathrm{Ca}^{2+}$ influx is reduced and this leads to an enhanced cell viability (Zhang et al., 2007). $\mathrm{H}_{2} \mathrm{O}_{2}$ and $\mathrm{TNF} \alpha$ stimulate TRPM2 tyrosine phosphorylation and the treatment with genistein, a non-selective tyrosine kinase inhibitor, and PP2, the Src kinase family inhibitor, abolishes the elevation of the intracellular calcium in TRPM2expressing cells, suggesting that a Src kinase family member is involved in the tyrosine phosphorylation of TRPM2. Moreover, using immunoprecipitation and glutathione-S transferase pull-down assays, Zhang et al. showed that PTPL1, a tyrosine phosphatase with implications in resistance to apoptosis and tumorigenesis (Yao et al., 2004; Abaan et al., 2005), interacts with TRPM2. Co-expression of TRPM2 and PTPL1 reduces TRPM2 tyrosine phosphorylation and inhibits the rise in intracellular calcium concentration and the loss of cell viability following $\mathrm{H}_{2} \mathrm{O}_{2}$ or TNF $\alpha$ treatment. In contrast, a reduction of endogenous PTPL1 expression induced by small interfering RNA determined an increase in TRPM2 
tyrosine phosphorylation, the elevation of intracellular calcium concentration and an increased susceptibility to cellular apoptosis. Thus, a mechanism that may intervene in cell death resistance is the modulation of TRPM2 channel tyrosine phosphorylation by PTPL1 (Zhang et al., 2007).

\section{TRPM7}

TRPM7 was first cloned by Runnels et al. which reported it as a protein that possesses kinase activity, but also ion channel activity (Runnels et al., 2001). TRPM7 contains in the $\mathrm{C}$-terminal region an active $\alpha$-kinase domain, which can phosphorylate itself (Runnels et al., 2001). TRPM7 functions as a homotetramer, is ubiquitously expressed, and is involved in cellular $\mathrm{Mg}^{2+}$ homeostasis (Nadler et al., 2001). In addition to $\mathrm{Mg}^{2+}$ transport across the membrane, TRPM7 also regulates $\mathrm{Ca}^{2+}$ influx in many cell types (Massullo et al., 2006). Transcripts of TRPM7 have been identified in rat brain microglia, as well as a current that has the hallmark features of expressed TRPM7 channels (Jiang et al., 2003). The Src kinase family appears to be involved in the modulation of TRPM7 in rat microglia. Activation of the current was inhibited by the broad-spectrum tyrosine kinase inhibitor genistein and by herbimycin A, a more selective Src kinase inhibitor. Moreover, Src40-58, a peptide that inhibits Src kinase function, had the same effect on TRPM7 current. TRPM7 is a channel permeable for $\mathrm{Ca}^{2+}$, therefore elevations in intracellular $\mathrm{Ca}^{2+}$ due to activation of TRPM7 might be important for microglia function (Jiang et al., 2003).

\section{TRPM8}

TRPM8 was cloned from prostate epithelial cells, where it was identified as a transcriptional marker (Tsavaler et al., 2001). Shortly after, the mouse TRPM8 was cloned from trigeminal ganglia and described as a cold-gated channel, activated by low temperatures, with a thermal threshold of approximately $25^{\circ} \mathrm{C}$ (McKemy et al., 2002). TRPM8 is a polymodal cation-permeable channel that is also activated by menthol (McKemy et al., 2002), icilin and WS-12 (Bodding et al., 2007) and voltage sensitive (Fernandez et al., 2011). TRPM8 is involved in several processes of cellular pathophysiology, including endothelial cell motility, proliferation, and protein unfolding (Noyer et al., 2018). These processes consist of signaling pathways that strictly control the proteins involved, for example through post-translational modifications, including tyrosine phosphorylation. The first hint of a possible tyrosine phosphorylation-induced modulation of TRPM8 was shown in a study where the Src kinase inhibitor PP2 exerted differential effects on TRPM8 and TRPA1 channels, expressed in SH-SY5Y cells, a human neuroblastoma line utilized as a neuronal cell model. WS-12-induced responses of TRPM8 were inhibited by PP2 pre-treatment in a dose-dependent manner, whereas PP3, an inactive analogue of PP2, did not affect responses to TRPM8 agonist. Interestingly, PP2 treatment had opposite effects on TRPA1, revealing a major difference in the modulation of TRPA1 and TRPM8 in SH-SY5Y cells (Morgan et al., 2014). We further explored this effect and recently demonstrated that TRPM8 can be modulated by Src-induced tyrosine phosphorylation (Manolache et al., 2020). HEK293T cells expressing human TRPM8 and treated with a protein phosphatase inhibitor, pervanadate, showed an increase in TRPM8 tyrosine phosphorylation. Src kinase induces tyrosine phosphorylation of human TRPM8 that can be inhibited by PP2 treatment. Furthermore, the phosphorylation and the cold-induced activation of TRPM8 are reduced after the inhibition of endogenous Src kinase. Experiments on cultured rat DRG neurons showed that TRPM8-mediated responses can be reduced by PP2 and dasatinib (another Src kinase inhibitor) treatment, and that effect can be successfully antagonized by pervanadate (Manolache et al., 2020).

\section{The Vanilloid Transient Receptor Potential ion channels}

The vanilloid subfamily of TRP channels was named after the first identified member, TRPV1, due to its activation induced by the vanilloid compound capsaicin (Caterina et al., 1997). TRPV subfamily comprises six members, TRPV1-TRPV6, which can be classified in two groups, TRPV1-4 and TRPV5-TRPV6. TRPV1-4 share about $45 \%$ structure identity (Vriens et al., 2004a), and are non-selective, $\mathrm{Ca}^{2+}$-permeable cation channels intrinsically sensitive to variations of temperature, from warm to hot temperatures, and activated by protons, osmotic strain, and certain exogenous chemicals (Caterina et al., 1997, 1999; Guler et al., 2002; Xu et al., 2002). TRPV1-4 are expressed in several cell types where they have essential roles in physiological processes, such as thermoregulation, osmoregulation, and nociception (Caterina et al., 2000; Liedtke and Friedman, 2003; Alessandri-Haber et al., 2003, 2004). TRPV6 and TRPV5 have greater sequence homology $(81 \%)$, present a high $\mathrm{Ca}^{2+}$-selectivity and are constitutively active under physiological conditions, which makes them important players in $\mathrm{Ca}^{2+}$ homeostasis (den Dekker et al., 2003). Currently, there are several studies which investigated the tyrosine phosphorylation and the modulation induced by this post-translational modification on TRPV1, TRPV2, TRPV4 and TRPV6 channels.

\section{TRPVI}

VR1 was first cloned from sensory neurons by the group of David Julius, and was reported to be activated by capsaicin, heat and protons and involved in pain signaling (Caterina et al., 1997). The channel name was later changed to TRPV1 (Montell et al., 2002). TRPV1 is a polymodal nonselective cation channel, with similar permeabilities for monovalent cations and a higher $\mathrm{Ca}^{2+}$ 
permeability (Caterina et al., 1997). In 2013, Liao et al. determined the structure of TRPV1 channel, using electron cryo-microscopy, which confirmed the previous structural studies (Liao et al., 2013). TRPV1 is formed by four identical monomers containing six transmembrane segments, with the $\mathrm{N}$ - and $\mathrm{C}$-terminal regions located intracellularly. The terminal regions and the intracellular loops between transmembrane segments contain phosphorylation sites for several kinases, which can modulate TRPV1 function. An investigation of the effect of tyrosine phosphorylation on TRPV1 channel function found that capsaicin-induced currents in DRG neurons were specifically inhibited by PP2, and increased by sodium orthovanadate, a tyrosine phosphatase inhibitor. In addition, PP2 specifically eliminated the currents of rat TRPV1-expressing HEK293 cells. Moreover, cotransfection of TRPV1 and the active form of Src kinase, v-Src, determined a fivefold increase in capsaicininduced currents, whereas co-transfection with dominantnegative c-Src resulted in a fourfold decrease of the currents. It was shown that Src kinase is associated with TRPV1, both in HEK293 cells and in rat DRG neurons, and that TRPV1 is tyrosine phosphorylated in cells transfected with both TRPV1 and v-Src (Jin et al., 2004). TRPV1-expressing nociceptors can be subjected to sensitization following injury or inflammation, a process mediated by the release of extracellular inflammatory mediators, such as bradykinin, adenosine triphosphate and nerve growth factor (NGF). A mechanism for NGFinduced sensitization of TRPV1 have been proposed by Zhang et al. They showed that NGF activates a signaling pathway which induces tyrosine phosphorylation of TRPV1 by Src kinase, leading to an upregulation of TRPV1 expression at the plasma membrane. The basal tyrosine phosphorylation and the NGF-induced increase of TRPV1 tyrosine phosphorylation was reduced by the specific Src kinase inhibitor PP2. Moreover, they identified tyrosine residue 200 (Y200) as the critical site for NGF-induced sensitization. An important remark is that the Y200 is conserved site in human, mouse, rat, guinea pig, rabbit, dog, and chicken TRPV1, which suggest that this residue may play an important role in TRPV1 regulation (Zhang et al., 2005). A protein involved in TRPV1 tyrosine dephosphorylation is Src homology 2 domain-containing tyrosine phosphatase 1 (Shp-1). Src-induced phosphorylation of TRPV1 was inhibited by co-transfection of Shp-1 and increased by co-transfection of Shp-1C455S, a dominant-negative form of Shp-1 (Zhang et al., 2005). Another study, focused on the modulation of TRPV1 by Shp-1, showed the co-expression and the direct interaction of Shp- 1 and TRPV1 in nociceptive DRG neurons (Xiao et al., 2015). Inhibition of Shp-1 increases TRPV1 tyrosinephosphorylation and induces thermal hyperalgesia in rats. Moreover, complete Freund's adjuvant-induced inflammatory pain in rats leads to an increase in the expression of Shp-1, which may be a mechanism of protection against excessive thermal hyperalgesia (Xiao et al., 2015).

\section{TRPV2}

TRPV2 was also identified by David Julius group as VRL-1, a TRPV1 homologue that is activated by high temperatures, with a threshold of $52^{\circ} \mathrm{C}$, but not by moderate heat, capsaicin, or protons (Caterina et al., 1999). TRPV2 channels are expressed in a subset of medium- to large-diameter neurons, but also in several non-neuronal tissues (Caterina et al., 1999). Repeated application of thermal stimuli can induce the sensitization of $\mathrm{C}$-fiber and $\mathrm{A} \delta$-fiber nociceptors, a phenomenon known as heat-heat sensitization (HHS). The ability of certain subclasses of nociceptors to exhibit HHS was investigated in a study which revealed that only nociceptors expressing TRPV2, or a combination of TRPV1 and TRPV2 exhibited HHS following repeated intense thermal stimulation. Moreover, it was shown that the process is calcium-dependent and that in cells coexpressing TRPV1 and TRPV2 the heat sensitivity was modulated through Ser/Thr phosphorylation or phospholipase A2 (PLA2)-dependent pathways, whereas in cells expressing only TRPV2, HHS may be linked to activation of tyrosine kinases. Application of genistein, a non-specific tyrosine kinase inhibitor, strongly reduced heat sensitization in TRPV2-expressing nociceptors, while inhibitors for PLA2 and Ser/Thr kinases had no effect. In contrast, in cells co-expressing TRPV1 and TRPV2, the sensitization was eliminated when PLA2 or Ser/Thr kinases were inhibited, raising the possibility that both pathways must be activated in order to produce HHS in a calcium-dependent manner. It was, therefore, concluded that TRPV2 plays a crucial role in the capacity of nociceptors to develop HHS (Rau et al., 2007).

\section{TRPV4}

TRPV4 was identified by Delany et al. as a mechanosensitive channel expressed in a large variety of tissues, including lung, heart, skin, brain, vascular endothelium and kidney, in the latter the expression being largely concentrated in the distal convoluted tubule (Delany et al., 2001; Xu et al., 2003). TRPV4 is a polymodal nonselective cation channel activated by the phorbol ester 4a-phorbol 12,13-didecanoate, acidic $\mathrm{pH}$ and temperatures over $27^{\circ} \mathrm{C}$ (Xu et al., 2003; Nilius et al., 2004; Vriens et al., 2004b).

$\mathrm{Xu}$ et al. described TRPV4 as being rapidly tyrosine phosphorylated in response to hypotonic stress both when heterologously expressed in HEK293 cells and in cultured native murine distal convoluted tubule cells. PP1, an inhibitor of the Src family of kinases, managed to inhibit TRPV4 phosphorylation in a dose-dependent manner and it was revealed that TRPV4 progressively associates with Src family kinases, particularly with Lyn and Yes, during hypotonic stress (Xu et al., 2003). 
Moreover, overexpression of wild type Lyn led to a dramatic potentiation of hypotonicity-dependent tyrosine phosphorylation of TRPV4, while transfection with dominant-negative Lyn inhibited the process modestly, but reproducibly. Tyrosine 253 (Y253) was identified as the site of tonicity-dependent tyrosine phosphorylation, which, when point mutated $(Y 253 F)$, eliminated the channel activity induced by hypotonicity. Taken together these results show that TRPV4 is phosphorylated at residue Y253 by the Src kinase, following hypotonic stress, which is necessary for channel function (Xu et al., 2003). However, it should be noted that other investigators were not able to reproduce these findings. They reported that application of a hypotonic solution to HEK293 cells expressing the TPRV4 Y253F mutant induces a strong rise in intracellular calcium, but not different to the one found in wild type TRPV4 expressing cells. They also demonstrated that pretreatment with PP1 and genistein did not alter hypotonicity-induced modifications in calcium concentration, in TRPV4expressing cells. Therefore, they concluded that phosphorylation of Y253 is not crucial for TRPV4 activation. Instead, they revealed that PLA2 activity is necessary for the activation of TRPV4 induced by cell swelling. Specifically, TRPV4 is activated via PLA2dependent production of arachidonic acid and its ulterior metabolization to 5',6'-epoxyeicosatrienoic acid by cytochrome P450 epoxygenase (Vriens et al., 2004b). Another team suggested that there may have been a selection bias in the study conducted by $\mathrm{Xu}$ et al. for the stable transfection of HEK293 cells with wild type TRPV4 or the $Y 253 F$ mutant. Therefore, in order to corroborate those findings in the absence of selection pressure, HEK293 cells were transiently transfected with the same constructs and calcium entry was monitored in response to hypotonicity. This experimental approach revealed that the cells transfected with TRPV4 exhibited a robust response to hypotonicity, while those transfected with the $Y 253 F$ mutant were almost completely insensitive to the same stimulus. It was concluded that differences between the findings of the two groups may have arisen because $\mathrm{Xu}$ et al. used stable transfection of a monocistronic expression vector and performed calcium assays using cells in suspension, whereas Vriens et al. transfected the cells transiently with a bicistronic vector for co-expression with a green-fluorescent protein and performed their assays on adherent cells (Xu et al., 2003; Vriens et al., 2004b; Cohen, 2005).

To further explore this issue, Wegierski et al. also examined the function of the Src family kinases-mediated tyrosine phosphorylation of TRPV4. They generated Madin-Darby Canine Kidney (MDCK) cell lines stably expressing mouse TRPV4 and identified phosphorylated tyrosine residues in purified proteins from these cells, while in cells pretreated with PP2, the phosphotyrosine signal was significantly diminished. A mass spectrometry analysis of the purified TRPV4 protein identified two phosphorylation sites, Y110 and Y805. In addition, an analysis of TRPV4 phosphorylation in HEK293T cells revealed a reduced Src-mediated phosphorylation of $Y 110 F$ and $Y 805 F$ TRPV4 variants, in comparison to wild type TRPV4. Moreover, cell treatment with $\mathrm{H}_{2} \mathrm{O}_{2}$ robustly increases TRPV4 tyrosine phosphorylation mediated by Src kinase, including the Y110 residue, suggesting that phosphorylation of this residue may be involved in the sensitization mechanism of TRPV4 channel in hyperalgesia (Wegierski et al., 2009).

Tyrosine phosphorylation also may be involved in TRPV4 modulation following activation of ProteaseActivated Receptor 2, known to play a role in the cellular response to injury and inflammation. The effect that human TRPV4 expression has on Protease-Activated Receptor 2-evoked calcium signaling was investigated in HEK293 cells and it was showed that the level of the sustained phase of the calcium response was proportional to TRPV4 expression. The effect of various kinase inhibitors was assessed: BIM-1, a protein kinase C inhibitor, had no effect on Protease-Activated Receptor 2-mediated signaling, whereas Src inhibitor 1 (Src1) substantially decreased the sustained phase of the response. Mutation of tyrosine residues $Y 110 F, Y 805 F$ and $Y 110 F / Y 805 F$ revealed similar transient increases in $\left[\mathrm{Ca}^{2+}\right]_{\mathrm{i}}$ in both wild type and mutant TRPV4 channels, while the sustained phase was significantly reduced in the Y110F mutant and slightly diminished in TRPV4 Y805F. Cells expressing the double mutant had a similar phenotype to cells expressing the single mutant TRPV4 Y110F (Poole et al., 2013).

Lastly, it was shown that in Human Umbilical Vein Endothelial Cells (HUVECs) and in HEK293 cells transfected with TRPV4, shear stress induces a rise in $\left[\mathrm{Ca}^{2+}\right] \mathrm{i}$, mostly determined by calcium entry via TRPV4, followed by translocation of TRPV4 channels to the plasma membrane via exocytosis within seconds. Substitution of tyrosine 110 (Y110) did not alter the activation of TRPV4 by its selective agonist GSK 1016790A, nor the shear stress-induced translocation of TRPV4 to the plasma membrane. However, it was clearly shown that shear stress did trigger phosphorylation of Y110, which was required for the TRPV4-mediated calcium response (Baratchi et al., 2016).

\section{TRPVG}

TRPV6 was initially described in rat small intestine (Peng et al., 1999) and not long after that, the human and mouse isoforms were also identified (Wissenbach et al., 2001; Hirnet et al., 2003). TRPV6 has the highest $\mathrm{Ca}^{2+}$ selectivity among TRP ion channels, and together with TRPV5, acts as a $\mathrm{Ca}^{2+}$ sensor, both having important roles in $\mathrm{Ca}^{2+}$ (re)absorption (Hirnet et al., 2003). TRPV6 is expressed in several cell types from the gastrointestinal tract, but also in lungs, placenta, prostate, and kidney, although the expression pattern differs between species (Hirnet et al., 2003). In addition, elevated levels of TRPV6 were found in subsets of human prostate cancer cells (Wissenbach et al., 2001). 
TRPV6 can be modulated via phosphorylation/dephosphorylation by Src kinase and phosphotyrosine phosphatase 1B protein (PTP1B), respectively (Sternfeld et al., 2005). It was initially shown that the regulation of TRPV6 by PTP1B is a store-dependent process. The inhibitor of PTP1B, bis-(N,N-dimethyl-hydroxamido) hydroxo-vanadate (DMHV), increased the plateau $\left[\mathrm{Ca}^{2+}\right]_{\mathrm{cyt}}$ within 45 minutes in TRPV6-expressing HEK293 cells, an effect which was absent in untransfected cells. Depletion of $\mathrm{Ca}^{2+}$ stores was a prerequisite for this effect, indicating that tyrosine phosphorylation modulated a key component of TRPV6mediated $\mathrm{Ca}^{2+}$ entry, or a regulatory protein involved in this process. PP1 treatment inhibited the DMHV-induced increase in $\mathrm{Ca}^{2+}$ entry mediated by TRPC6, proving that tyrosine phosphorylation is responsible for the DMHV effect. Phosphorylation of the channel itself was evaluated and the investigators found that co-expression of TRPC6 and the tyrosine kinase Src did not determined the phosphorylation of TRPV6 in control conditions, but that pervanadate treatment led to a weak tyrosine phosphorylation (Sternfeld et al., 2005). In another study, the same group aimed to identify the specific tyrosine residues which mediate these effects. They found that both exogenous Fyn and endogenous Src kinases could mediate the channel's phosphorylation and identified PTP1B as the phosphatase interacting with TRPV6. It was observed that the PTP1B phosphatase binds to TRPV6 channel in the $\mathrm{N}$-terminus region which includes the first 191 amino acids. They also showed that tyrosine phosphorylation of this region is critical for the DMHVinduced calcium entry. Furthermore, it was determined that mutations of either Y161 or Y162 did not modify TRPV6 phosphorylation or the effect that DMHV has on calcium influx. However, when both residues were substituted, tyrosine phosphorylation was absent, even though the interaction with PTP1B was not modified. In conclusion, both Y161 and Y162 play a critical role in the regulation of TRPV6 by tyrosine phosphorylation and its functional consequences (Sternfeld et al., 2007). Single mutations of either Y161 or Y162 did not alter phosphorylation of the channel or the effect that DMHV has on calcium influx.

\section{Conclusions}

In this review article we described the biophysical and physiological consequences following tyrosine phosphorylation of certain non-selective cation channels from the TRP channel superfamily. Interestingly, in most of these cases it appears that tyrosine Phosphorylation promotes channel function and leads to increased activity or functional expression in the plasma membrane. Among other cellular functions, TRP channel tyrosine phosphorylation has been associated with increased storeoperated calcium entry (via modulation of TRPC4), calcium accumulation in podocytes (TRPC6), induction of apoptotic pathways (TRPM2), microglia activation
(TRPM7), hypersensitivity to cold (TRPM8) and inflammatory heat hyperalgesia (TRPV1). In addition to their physiological role in sensing the extracellular or internal milieu of various cell types, certain TRP channels have also been recently implicated in the pathophysiology of tumorigenesis, cell proliferation and migration, and therefore it can be safely assumed that tyrosine phosphorylation of these channels may be involved in cancer pathophysiology. Taken together, the results reviewed in this paper indicate that tyrosine phosphorylation is a key modulatory feature of TRP channels, is involved in major physiological and pathophysiological signaling pathways and could be a valid target for clinical intervention and molecular therapies.

\section{Acknowledgments}

This work was supported by a grant of the Romanian Ministry of Education and Research, CCCDI-UEFISCDI, PN-III-P2-2.1-PED-2019-5179, within PNCDI III.

(C)The Author(s) 2020

Open Access This article is distributed under the terms of the Creative Commons Attribution 4.0 International License (http://creativecommons.org/licenses/by/4.0/) which permits unrestricted use, distribution, and reproduction in any medium, provided you give appropriate credit to the original author(s) and the source, provide a link to the Creative Commons license, and indicate if changes were made.

\section{References}

Aarts M., Iihara K., Wei W.L., Xiong Z.G., Arundine M., Cerwinski W., Macdonald J.F., Tymianski M. 2003. A key role for TRPM7 channels in anoxic neuronal death. Cell 115, 863-877.

Abaan O.D., Levenson A., Khan O., Furth P.A., Uren A., Toretsky J.A. 2005. PTPL1 is a direct transcriptional target of EWS-FLI1 and modulates Ewing's Sarcoma tumorigenesis. Oncogene 24, 2715-2722.

Alessandri-Haber N., Dina O.A., Yeh J.J., Parada C.A., Reichling D.B., Levine J.D. 2004. Transient receptor potential vanilloid 4 is essential in chemotherapyinduced neuropathic pain in the rat. J Neurosci. 24, 4444-4452.

Alessandri-Haber N., Yeh J.J., Boyd A.E., Parada C.A., Chen X., Reichling D.B., Levine J.D. 2003. Hypotonicity induces TRPV4-mediated nociception in rat. Neuron 39, 497-511.

Anderson M., Roshanravan H., Khine J., Dryer S.E. 2014. Angiotensin II activation of TRPC6 channels in rat podocytes requires generation of reactive oxygen species. J Cell Physiol. 229, 434-442.

Andersson D.A., Gentry C., Moss S., Bevan S. 2008. Transient receptor potential A1 is a sensory receptor 
for multiple products of oxidative stress. J Neurosci. 28, 2485-2494.

Baratchi S., Almazi J.G., Darby W., Tovar-Lopez F.J., Mitchell A., Mcintyre P. 2016. Shear stress mediates exocytosis of functional TRPV4 channels in endothelial cells. Cell Mol Life Sci. 73, 649-666.

Birnbaumer L., Zhu X., Jiang M., Boulay G., Peyton M., Vannier B., Brown D., Platano D., Sadeghi H., Stefani E., Birnbaumer M. 1996. On the molecular basis and regulation of cellular capacitative calcium entry: roles for Trp proteins. Proc Natl Acad Sci USA. 93, 15195-15202.

Bodding M., Wissenbach U., Flockerzi V. 2007. Characterisation of TRPM8 as a pharmacophore receptor. Cell Calcium 42, 618-628.

Boulay G., Zhu X., Peyton M., Jiang M., Hurst R., Stefani E., Birnbaumer L. 1997. Cloning and expression of a novel mammalian homolog of Drosophila transient receptor potential (Trp) involved in calcium entry secondary to activation of receptors coupled by the $\mathrm{Gq}$ class of $\mathrm{G}$ protein. J Biol Chem. 272, 29672-29680.

Caterina M.J., Leffler A., Malmberg A.B., Martin W.J., Trafton J., Petersen-Zeitz K.R., Koltzenburg M., Basbaum A.I., Julius D. 2000. Impaired nociception and pain sensation in mice lacking the capsaicin receptor. Science 288, 306-313.

Caterina M.J., Rosen T.A., Tominaga M., Brake A.J., Julius, D. 1999. A capsaicin-receptor homologue with a high threshold for noxious heat. Nature 398, 436441.

Caterina M.J., Schumacher M.A., Tominaga M., Rosen T.A., Levine J.D., Julius, D. 1997. The capsaicin receptor: a heat-activated ion channel in the pain pathway. Nature 389, 816-824.

Clapham D.E., Runnels L.W., Strubing C. 2001. The TRP ion channel family. Nat Rev Neurosci. 2, $387-$ 396.

Cohen D.M. 2005. TRPV4 and the mammalian kidney. Pflugers Arch. 451, 168-175.

Cohen M.R., Moiseenkova-Bell, V.Y. 2014. Structure of thermally activated TRP channels. Curr Top Membr. 74, 181-211.

Delany N.S., Hurle M., Facer P., Alnadaf T., Plumpton C., Kinghorn I., See C.G., Costigan M., Anand P., Woolf C.J., Crowther D., Sanseau P., Tate S.N. 2001. Identification and characterization of a novel human vanilloid receptor-like protein, VRL-2. Physiol Genomics. 4, 165-174.

Den Dekker E., Hoenderop J.G., Nilius B., Bindels R.J. 2003. The epithelial calcium channels, TRPV5, TRPV6: from identification towards regulation. Cell Calcium 33, 497-507.

Duncan L.M., Deeds J., Hunter J., Shao J., Holmgren L.M., Woolf E.A., Tepper R.I., Shyjan A.W. 1998. Down-regulation of the novel gene melastatin correlates with potential for melanoma metastasis. Cancer Res. 58, 1515-1520.
Fernandez J.A., Skryma R., Bidaux G., Magleby K.L., Scholfield C.N., Mcgeown J.G., Prevarskaya N., Zholos A.V. 2011. Voltage- and cold-dependent gating of single TRPM8 ion channels. J Gen Physiol. 137, 173-195.

Fonfria E., Marshall I.C., Boyfield I., Skaper S.D., Hughes J.P., Owen D.E., Zhang W., Miller B.A., Benham C.D., Mcnulty S. 2005. Amyloid betapeptide(1-42) and hydrogen peroxide-induced toxicity are mediated by TRPM2 in rat primary striatal cultures. J Neurochem. 95, 715-723.

Freichel M., Suh S.H., Pfeifer A., Schweig U., Trost C., Weissgerber P., Biel M., Philipp S., Freise D., Droogmans G., Hofmann F., Flockerzi V., Nilius B. 2001. Lack of an endothelial store-operated $\mathrm{Ca} 2+$ current impairs agonist-dependent vasorelaxation in TRP4-/- mice. Nat Cell Biol. 3, 121-127.

Gasser A., Glassmeier G., Fliegert R., Langhorst M.F., Meinke S., Hein D., Kruger S., Weber K., Heiner I., Oppenheimer N., Schwarz J.R., Guse A.H. 2006. Activation of $\mathrm{T}$ cell calcium influx by the second messenger ADP-ribose. J Biol Chem. 281, 2489-2496.

Guinamard R., Salle L., Simard C. 2011. The nonselective monovalent cationic channels TRPM4 and TRPM5. Adv Exp Med Biol. 704, 147-171.

Guler A.D., Lee H., Iida T., Shimizu I., Tominaga M., Caterina M. 2002. Heat-evoked activation of the ion channel, TRPV4. J Neurosci. 22, 6408-6414.

Gutkind J.S., Robbins K.C. 1992. Activation of transforming $G$ protein-coupled receptors induces rapid tyrosine phosphorylation of cellular proteins, including p125FAK and the p130 v-src substrate. Biochem Biophys Res Commun. 188, 155-161.

Hara Y., Wakamori M., Ishii M., Maeno E., Nishida M., Yoshida T., Yamada H., Shimizu S., Mori E., Kudoh J., Shimizu N., Kurose H., Okada Y., Imoto K., Mori Y. 2002. LTRPC2 Ca2+-permeable channel activated by changes in redox status confers susceptibility to cell death. Mol Cell. 9, 163-173.

Harteneck C., Plant T.D., Schultz G. 2000. From worm to man: three subfamilies of TRP channels. Trends Neurosci. 23, 159-166.

Hirnet D., Olausson J., Fecher-Trost C., Bodding M., Nastainczyk W., Wissenbach U., Flockerzi V., Freichel M. 2003. The TRPV6 gene, cDNA and protein. Cell Calcium 33, 509-518.

Hisatsune C., Kuroda Y., Nakamura K., Inoue T., Nakamura T., Michikawa T., Mizutani A., Mikoshiba K. 2004. Regulation of TRPC6 channel activity by tyrosine phosphorylation. J Biol Chem. 279, 1888718894.

Hofmann T., Obukhov A.G., Schaefer M., Harteneck C., Gudermann T., Schultz G. 1999. Direct activation of human TRPC6 and TRPC3 channels by diacylglycerol. Nature 397, 259-263.

Hofmann T., Schaefer M., Schultz G., Gudermann T. 2002. Subunit composition of mammalian transient 
receptor potential channels in living cells. Proc Natl Acad Sci U S A. 99, 7461-7466.

Igishi T., Gutkind J. S. 1998. Tyrosine kinases of the Src family participate in signaling to MAP kinase from both $\mathrm{Gq}$ and $\mathrm{Gi}$-coupled receptors. Biochem Biophys Res Commun. 244, 5-10.

Jiang X., Newell E.W., Schlichter L.C. 2003. Regulation of a TRPM7-like current in rat brain microglia. J Biol Chem. 278, 42867-42876.

Jin X., Morsy N., Winston J., Pasricha P.J., Garrett K., Akbarali H.I. 2004. Modulation of TRPV1 by nonreceptor tyrosine kinase, c-Src kinase. $A m J$ Physiol Cell Physiol. 287, C558-563.

Kanda S., Harita Y., Shibagaki Y., Sekine T., Igarashi T., Inoue T., Hattori S. 2011. Tyrosine phosphorylationdependent activation of TRPC6 regulated by PLCgamma1 and nephrin: effect of mutations associated with focal segmental glomerulosclerosis. Mol Biol Cell. 22, 1824-1835.

Kawasaki B.T., Liao Y., Birnbaumer L. 2006. Role of Src in C3 transient receptor potential channel function and evidence for a heterogeneous makeup of receptorand store-operated $\mathrm{Ca} 2+$ entry channels. Proc Natl Acad Sci U S A. 103, 335-340.

Kim E.Y., Anderson M., Dryer S.E. 2012. Insulin increases surface expression of TRPC6 channels in podocytes: role of NADPH oxidases and reactive oxygen species. Am J Physiol Renal Physiol. 302, F298-307.

Kim E.Y., Hassanzadeh Khayyat N., Dryer S.E. 2018. Mechanisms underlying modulation of podocyte TRPC6 channels by suPAR: Role of NADPH oxidases and Src family tyrosine kinases. Biochim Biophys Acta Mol Basis Dis. 1864, 3527-3536.

Li M., Jiang J., Yue L. 2006. Functional characterization of homo- and heteromeric channel kinases TRPM6 and TRPM7. J Gen Physiol. 127, 525-537.

Liao M., Cao E., Julius D., Cheng Y. 2013. Structure of the TRPV1 ion channel determined by electron cryomicroscopy. Nature 504, 107-112.

Liedtke W., Friedman J.M. 2003. Abnormal osmotic regulation in trpv4-/- mice. Proc Natl Acad Sci U S A. 100, 13698-13703.

Liman E.R., Innan H. 2003. Relaxed selective pressure on an essential component of pheromone transduction in primate evolution. Proc Natl Acad Sci U S A. 100, 3328-3332.

Liu D., Liman E.R. 2003. Intracellular $\mathrm{Ca} 2+$ and the phospholipid PIP2 regulate the taste transduction ion channel TRPM5. Proc Natl Acad Sci U S A. 100, 15160-15165.

Liu X., Yao X., Tsang S.Y. 2020. Post-Translational Modification and Natural Mutation of TRPC Channels. Cells 9, 135.

Manolache A., Selescu T., Maier G.L., Mentel M., Ionescu A.E., Neacsu C., Babes A., Szedlacsek S.E. 2020. Regulation of TRPM8 channel activity by Src- mediated tyrosine phosphorylation. J Cell Physiol. 235, 5192-5203.

Massullo P., Sumoza-Toledo A., Bhagat H., PartidaSanchez S. 2006. TRPM channels, calcium and redox sensors during innate immune responses. Semin Cell Dev Biol. 17, 654-666.

Mckemy D.D., Neuhausser W.M., Julius D. 2002. Identification of a cold receptor reveals a general role for TRP channels in thermosensation. Nature 416, 5258.

Mery L., Strauss B., Dufour J.F., Krause K.H., Hoth M. 2002. The PDZ-interacting domain of TRPC4 controls its localization and surface expression in HEK293 cells. J Cell Sci. 115, 3497-3508.

Montell C. 2003. $\mathrm{Mg} 2+$ homeostasis: the $\mathrm{Mg} 2+$ nificent TRPM chanzymes. Curr Biol. 13, R799-801.

Montell C., Birnbaumer L., Flockerzi V., Bindels R.J., Bruford E.A., Caterina M.J., Clapham D.E., Harteneck C., Heller S., Julius D., Kojima I., Mori Y., Penner R., Prawitt D., Scharenberg A.M., Schultz G., Shimizu N., Zhu M.X. 2002. A unified nomenclature for the superfamily of TRP cation channels. Mol Cell. 9, 229-231.

Morgan K., Sadofsky L.R., Crow C., Morice A.H. 2014. Human TRPM8 and TRPA1 pain channels, including a gene variant with increased sensitivity to agonists (TRPA1 R797T), exhibit differential regulation by SRC-tyrosine kinase inhibitor. Biosci Rep. 34.

Nadler M.J., Hermosura M.C., Inabe K., Perraud A.L., Zhu Q., Stokes A.J., Kurosaki T., Kinet J.P., Penner R., Scharenberg A.M., Fleig A. 2001. LTRPC7 is a Mg.ATP-regulated divalent cation channel required for cell viability. Nature 411, 590-595.

Nilius B., Vriens J., Prenen J., Droogmans G., Voets T. 2004. TRPV4 calcium entry channel: a paradigm for gating diversity. Am J Physiol Cell Physiol. 286, C195-205.

Noyer L., Grolez G.P., Prevarskaya N., Gkika D., Lemonnier L. 2018. TRPM8 and prostate: a cold case? Pflugers Arch. 470, 1419-1429.

Odell A.F., Scott J.L., Van Helden D.F. 2005. Epidermal growth factor induces tyrosine phosphorylation, membrane insertion, and activation of transient receptor potential channel 4. J Biol Chem. 280, 37974-37987.

Okada T., Inoue R., Yamazaki K., Maeda A., Kurosaki T., Yamakuni T., Tanaka I., Shimizu S., Ikenaka K., Imoto K., Mori Y. 1999. Molecular and functional characterization of a novel mouse transient receptor potential protein homologue TRP7. $\mathrm{Ca}(2+)$-permeable cation channel that is constitutively activated and enhanced by stimulation of $G$ protein-coupled receptor. J Biol Chem. 274, 27359-27370.

Peng J.B., Chen X.Z., Berger U.V., Vassilev P.M., Tsukaguchi H., Brown E.M., Hediger M.A. 1999. Molecular cloning and characterization of a channellike transporter mediating intestinal calcium absorption. J Biol Chem. 274, 22739-22746. 
Philipp S., Cavalie A., Freichel M., Wissenbach U., Zimmer S., Trost C., Marquart A., Murakami M., Flockerzi V. 1996. A mammalian capacitative calcium entry channel homologous to Drosophila TRP and TRPL. EMBO J. 15, 6166-6171.

Poole D.P., Amadesi S., Veldhuis N.A., Abogadie F.C., Lieu T., Darby W., Liedtke W., Lew M.J., Mcintyre P., Bunnett N.W. 2013. Protease-activated receptor 2 (PAR2) protein and transient receptor potential vanilloid 4 (TRPV4) protein coupling is required for sustained inflammatory signaling. J Biol Chem. 288, 5790-5802.

Prawitt D., Enklaar T., Klemm G., Gartner B., Spangenberg C., Winterpacht A., Higgins M., Pelletier J., Zabel B. 2000. Identification and characterization of MTR1, a novel gene with homology to melastatin (MLSN1) and the trp gene family located in the BWS-WT2 critical region on chromosome 11p15.5 and showing allele-specific expression. Hum Mol Genet. 9, 203-216.

Rau K.K., Jiang N., Johnson R.D., Cooper B.Y. 2007. Heat sensitization in skin and muscle nociceptors expressing distinct combinations of TRPV1 and TRPV2 protein. J Neurophysiol. 97, 2651-2662.

Runnels L.W., Yue L., Clapham D.E. 2001. TRP-PLIK, a bifunctional protein with kinase and ion channel activities. Science 291, 1043-1047.

Sano Y., Inamura K., Miyake A., Mochizuki S., Yokoi H., Matsushime H., Furuichi K. 2001. Immunocyte $\mathrm{Ca} 2+$ influx system mediated by LTRPC2. Science 293, 1327-1330.

Schaefer M., Plant T.D., Obukhov A.G., Hofmann T., Gudermann T., Schultz G. 2000. Receptor-mediated regulation of the nonselective cation channels TRPC4 and TRPC5. J Biol Chem. 275, 17517-17526.

Sternfeld L., Anderie I., Schmid A., Al-Shaldi H., Krause E., Magg T., Schreiner D., Hofer H.W., Schulz I. 2007. Identification of tyrosines in the putative regulatory site of the $\mathrm{Ca}^{2+}$ channel TRPV6. Cell Calcium 42, 91-102.

Sternfeld L., Krause E., Schmid A., Anderie I., Latas A., Al-Shaldi H., Kohl A., Evers K., Hofer H.W., Schulz I. 2005. Tyrosine phosphatase PTP1B interacts with TRPV6 in vivo and plays a role in TRPV6-mediated calcium influx in HEK293 cells. Cell Signal. 17, 951960.

Tsavaler L., Shapero M.H., Morkowski S., Laus R. 2001. Trp-p8, a novel prostate-specific gene, is up-regulated in prostate cancer and other malignancies and shares high homology with transient receptor potential calcium channel proteins. Cancer Res. 61, 3760-3769.

Vannier B., Zhu X., Brown D., Birnbaumer L. 1998. The membrane topology of human transient receptor potential 3 as inferred from glycosylation-scanning mutagenesis and epitope immunocytochemistry. $J$ Biol Chem. 273, 8675-8679.
Vazquez G., Wedel B.J., Aziz O., Trebak M., Putney J.W.Jr. 2004a. The mammalian TRPC cation channels. Biochim Biophys Acta. 1742, 21-36.

Vazquez G., Wedel B.J., Kawasaki B.T., Bird G.S., Putney J.W.Jr. 2004b. Obligatory role of Src kinase in the signaling mechanism for TRPC 3 cation channels. J Biol Chem. 279, 40521-40528.

Vennekens R., Hoenderop J.G., Prenen J., Stuiver M., Willems P.H., Droogmans, G., Nilius, B. \& Bindels, R. J. 2000. Permeation and gating properties of the novel epithelial $\mathrm{Ca}(2+)$ channel. J Biol Chem. 275, 3963-3969.

Vriens J., Owsianik G., Voets T., Droogmans G., Nilius B. 2004a. Invertebrate TRP proteins as functional models for mammalian channels. Pflugers Arch. 449, 213-226.

Vriens J., Watanabe H., Janssens A., Droogmans G., Voets T., Nilius B. 2004b. Cell swelling, heat, and chemical agonists use distinct pathways for the activation of the cation channel TRPV4. Proc Natl Acad Sci U S A. 101, 396-401.

Wegierski T., Lewandrowski U., Muller B., Sickmann A., Walz G. 2009. Tyrosine phosphorylation modulates the activity of TRPV4 in response to defined stimuli. J Biol Chem. 284, 2923-2933.

Wehage E., Eisfeld J., Heiner I., Jungling E., Zitt C., Luckhoff A. 2002. Activation of the cation channel long transient receptor potential channel 2 (LTRPC2) by hydrogen peroxide. A splice variant reveals a mode of activation independent of ADP-ribose. $J$ Biol Chem. 277, 23150-23156.

Wissenbach U., Niemeyer B.A., Fixemer T., Schneidewind A., Trost C., Cavalie A., Reus K., Meese E., Bonkhoff H., Flockerzi V. 2001. Expression of CaT-like, a novel calcium-selective channel, correlates with the malignancy of prostate cancer. J Biol Chem. 276, 19461-19468.

Xiao X., Zhao X.T., Xu L.C., Yue L.P., Liu F.Y., Cai J., Liao F.F., Kong J.G., Xing G.G., Yi M., Wan Y. 2015. Shp-1 dephosphorylates TRPV1 in dorsal root ganglion neurons and alleviates CFA-induced inflammatory pain in rats. Pain 156, 597-608.

Xu H., Ramsey I.S., Kotecha S.A., Moran M.M., Chong J.A., Lawson D., Ge P., Lilly J., Silos-Santiago I., Xie Y., Distefano P.S., Curtis R., Clapham D.E. 2002. TRPV3 is a calcium-permeable temperature-sensitive cation channel. Nature 418, 181-186.

Xu H., Zhao H., Tian W., Yoshida K., Roullet J.B., Cohen D.M. 2003. Regulation of a transient receptor potential (TRP) channel by tyrosine phosphorylation. SRC family kinase-dependent tyrosine phosphorylation of TRPV4 on TYR-253 mediates its response to hypotonic stress. J Biol Chem. 278, 11520-11527.

Yang H., Mergler S., Sun X., Wang Z., Lu L., Bonanno J.A., Pleyer U., Reinach P.S. 2005. TRPC4 knockdown suppresses epidermal growth factorinduced store-operated channel activation and growth 
in human corneal epithelial cells. J Biol Chem. 280, 32230-32237.

Yao H., Song E., Chen J., Hamar P. 2004. Expression of FAP-1 by human colon adenocarcinoma: implication for resistance against Fas-mediated apoptosis in cancer. Br J Cancer. 91, 1718-1725.

Yu L., Lin Q., Liao H., Feng J., Dong X., Ye J. 2010. TGF-beta1 induces podocyte injury through Smad3ERK-NF-kappaB pathway and Fyn-dependent TRPC6 phosphorylation. Cell Physiol Biochem. 26, 869-878.

Zhang W., Hirschler-Laszkiewicz I., Tong Q., Conrad K., Sun S.C., Penn L., Barber D.L., Stahl R., Carey D.J., Cheung J.Y., Miller B.A. 2006. TRPM2 is an ion channel that modulates hematopoietic cell death through activation of caspases and PARP cleavage. Am J Physiol Cell Physiol. 290, C1146-1159.

Zhang W., Tong Q., Conrad K., Wozney J., Cheung J.Y., Miller B.A. 2007. Regulation of TRP channel TRPM2 by the tyrosine phosphatase PTPL1. Am J Physiol Cell Physiol. 292, C1746-1758.

Zhang X., Huang J., Mcnaughton P.A. 2005. NGF rapidly increases membrane expression of TRPV1 heat-gated ion channels. The EMBO Journal 24, 4211-4223.

Zholos A., Johnson C., Burdyga T., Melanaphy D. 2011. TRPM channels in the vasculature. Adv Exp Med Biol. 704, 707-729.

Zhu X., Jiang M., Peyton M., Boulay G., Hurst R., Stefani E., Birnbaumer L. 1996. trp, a novel mammalian gene family essential for agonistactivated capacitative $\mathrm{Ca}^{2+}$ entry. Cell85, 661-671. 